HELMINTHOLOGIA, 55, 1: 21 - 32, 2018

\title{
Complementary effect of Capparis spinosa L. and silymarin with/without praziquantel on mice experimentally infected with Schistosoma mansoni
}

\author{
S. S. EL-HAWARY' ${ }^{1}$ K. F. TAHA ${ }^{2}$, F. N. KIRILLOS 1 , A. A. DAHAB ${ }^{3}$, A. A. EL-MAHIS ${ }^{2}$, S. H. EL-SAYED ${ }^{4,5 *}$
}

\begin{abstract}
${ }^{1}$ Pharmacognosy Department, Faculty of Pharmacy, Cairo University, Cairo, Egypt; ${ }^{2}$ Applied Research Center of Medicinal Plants, National Organization of Drug Control and Research, Cairo, Egypt; ${ }^{3}$ Department of Medicinal and Aromatic Plants, Horticulture Research Institute, ARC, Cairo, Egypt; ${ }^{4}$ Medical Parasitology Department, Theodor Bilharz Research Institute, Imbaba,

Giza, Egypt; ${ }^{5}$ Medical Parasitology Department, Faculty of Medicne, Helwan University, Cairo, Egypt, *E-mail: dr_sh_helmy@yahoo.com, shaimaahelmy13@gmail.com
\end{abstract}

Article info

Received August 17, 2017 Accepted November 20, 2017

\section{Summary}

Schistosomiasis remains to be the most common fibrotic disease resulting from inflammation and deposition of scar tissue around trapped parasitic eggs in the liver. Though chemotherapy eradicates matured worms efficiently and prevents the accumulation of schistosome eggs, fewer effective drugs are directed to reverse the present hepatic fibrosis. Therefore, treatment targeting hepatic fibrosis associated with schistosomiasis remains a challenging proposition.

The present study was designed to investigate the potential complementary schistosomicidal and hepatoprotective activities of the methanol extract of Capparis spinosa L. (C. spinosa) with or without praziquantel (PZQ) and compare results with silymarin (Milk thistle), a known hepatoprotective and antifibrotic agent, on induced liver fibrosis by experimental Schistosoma mansoni (S. mansoni) infection. Total polyphenols in the extract were determined using colorimetric assay.

C. spinosa L. caused a partial decrease in worm burden; a statistically significant reduction in hepatic and intestinal tissue egg load, what was associated histopathologically with decreasing in both the number and diameter of granulomas, as well as restoring serum aminotransferases (AST \& ALT), alkaline phosphatase (ALP) and improving liver albumin synthesis. The best results were obtained in the group of mice treated with C. spinosa L. and PZQ together. Quantitative estimation of total polyphenols content using colorimetric assay showed that $C$. spinosa $L$. leaves contain higher concentration of polyphenolic compounds than fruits.

It was concluded that $C$. spinosa $L$. has a promising hepatoprotective and antifibrotic properties and could be introduced as a safe and effective therapeutic tool with PZQ in the treatment of schistosomal liver fibrosis. Nevertheless further studies on the mechanism of action of $C$. spinosa $L$. in chronic liver diseases may shed light on developing therapeutic methods in clinical practice.

Keywords: Schistosoma mansoni; Capparis spinosa L.; praziquantel; silymarin; hepatoprotective; colorimetric assay

\section{Introduction}

Schistosomiasis produced by S. mansoni continues to be an essential cause of parasitic morbidity and mortality worldwide and is the most common fibrotic disease due to inflammation and the deposition of scar tissue around parasitic eggs trapped in the liver (Burke et al., 2010). This disease is the second most important tropical disease after malaria and has significant impact on public health and socioeconomic problems in developing countries. (Abdel Aziz et al., 2011).

\footnotetext{
* - corresponding author
} 
It is known that fibrosis may be reversible while cirrhosis is an irreversible pathological process, Therefore it is important to prevent the progression of fibrosis to cirrhosis. However, there is no effective anti-fibrotic drug to date and the efficacy of most treatments were not conclusive in clinical trials (Bataller \& Brenner, 2005). Currently available treatment for many liver diseases is ineffective and is associated with many adverse effects, usually resulting in liver transplantation as the only choice of treatment. Novel compounds which prevent or halt scarring response are urgently needed to prevent the progression to end-stage liver disease (Albanis et al., 2003).

Praziquantel (PZQ), as a safe anti-schistosomal drug, has been used for more than 30 years (Fenwick et al., 2003). In general, specific treatment of schistosomiasis results in parasite elimination, and later on, a slight reduction in hepatic fibrosis that is attributed to parasite eradication (Homeida et al., 1991). However, loss of $P Z Q$ efficacy in schistosomiasis treatment was reported from Egypt, where the drug was being used aggressively (Ali, 2011). Returning to nature was one of the suggested solutions for treatment of liver damage resulting from schistosomiasis. Referring to traditional medicine it was found that $C$. spinosa $L$. (Caper) a wild plant growing in Egyptian deserts was used by the Ancient Egyptians in the treatment of liver diseases (Tlili et al., 2011). Several claims about the usefulness of $C$. spinosa and its low toxicity were justified by experimental evidences. It was known by its anthelmintic activity in traditional Arab medicine and used to treat liver dysfunction. In Ayurvedeic medicine capers are recorded as hepatic stimulants and protectors, thus improving liver function (Sher \& Alyemeni, 2010). Its extract is one of the constituents of Ayurvedeic preparation administered to treat preliminary cases of acute viral hepatitis and cirrhosis of liver and has shown encouraging results against viral infection. Bedouin heat leaves in butter and used this preparation against external parasitic disease of camel (Batanouny, 2005), for all these reasons C. spinosa L. was the plant of primary choice for this study.

C. spinosa $\mathrm{L}$. is a perennial spiny bush that bears rounded, fleshy leaves and big white to pinkish-white flowers (Manikandaselvi \& Brindha, 2014). Several biological activities and medicinal properties of C. spinosa L. were scientifically proved, it has anthelmintic, cytotoxic, anti-inflammatory (Al-Said et al.,1988; Zhou et al., 2011), antiarthritic (Feng et al., 2011), antioxidant (Tesoriere et al., 2007; Bouriche et al., 2011), antibacterial (Sherif et al., 2013), antifungal (Lam et al., 2008), antidiabetic (Rahmani et al., 2014), antiviral, immunomodulatory (Arena et al., 2008), anticarcinogenic (Lam et al., 2008; Kulisic-Bilusic et al., 2012; Sherif et al., 2013), antihistaminic, chondroprotective, (Sher \& Alyemeni, 2010), cardiovascular, hypolipidemic, antiallergic (Manikandaselvi et al., 2016) and antihepatotoxic activities (Gadgoli \& Mishra, 1999; Satyanarayana et al., 2009; Bigoniya et al., 2013)

There is still intensive need for effective treatment of schistosomal liver fibrosis with minimal side effects. Thus, it was worthy to investigate the biological effect of $C$. spinosa $L$. which is one of the plants known in Ancient Egyptians Arab, Iranian, Unani, Chinese and Ayurvedic medicines. In the hope of finding a complementary natural product that has hepatoprotective and antifibrotic activity which support and benefit in the therapy of schistosomiasis. The present study, therefore, suggest full sustainable exploitation of C. spinosa with the aim to benefit Egypt from its flora and validate the use of this plant for the first time. Either alone or combined with the antischistosomal $P Z Q$ on schistosomal liver fibrosis and then comparing the results with silymarin, a known hepatoprotective and antifibrotic agent.

\section{Material and Methods}

\section{Plant material}

Leaves and fruits of $C$. spinosa L. were collected from Agiba in Marsa Matroh, Egypt, May 2016. Identification of the plants was kindly confirmed by Mrs. Threase Labib, senior botanist of plant taxonomy at El-Orman botanical garden, Cairo, Egypt. Voucher specimens No. (362014) has been deposited in the herbarium of Faculty of Pharmacy, Cairo University.

\section{Preparation of methanol extract}

A sample of $C$. spinosa $L$. leaves were separately air dried in the shade, powdered and kept in tightly closed amber colored glass container. $100 \mathrm{gm}$ of dried leaves was extracted in methanol, then sonicated for 1 hour in an ultrasound bath, kept overnight and then filtered. The clear filtrate was freeze dried (El-Hawary et al., 2015).

\section{Quantitative estimation of total polyphenols using colorimetric assay}

Assay of total polyphenols was done using UV-visible spectrophotometer, Nicolet evolution 100 (Thermo) was used for recording UV spectra and measuring the absorbance in UV range.

\section{Establishment of the calibration curve and sample preparation}

A calibration curve was prepared using gallic acid as a standard at concentrations of $5,10,20,30,40,50,60,70,80$, and $90 \mu \mathrm{g} /$ $\mathrm{ml}$ in methanol.

Powdered air-dried samples of both leaves and fruit of $C$. spinosa L. were separately extracted, till exhaustion, with methanol. The solvent was evaporated to dryness. Stock solution $(25 \mathrm{mg} \%$ in methanol) of leaves and fruits extracts were prepared.

The method adopted was based on measuring the intensity of the blue color developed when phenolic compounds make a complex with Folin-Ciocalteau reagent using gallic acid as a standard. The concentration of the total phenolics was calculated as gallic acid equivalent (GAE) with reference to a pre-established standard calibration curve. Folin-Ciocalteau reagent (100 $\mu$ l) was added to a test tube containing $20 \mu \mathrm{l}$ of the extract solution. The contents were mixed, and a saturated aqueous sodium carbonate solution $(200 \mu \mathrm{l})$ was added. The volume was adjusted to $2 \mathrm{ml}$ by the addition of $0.68 \mathrm{ml}$ of milliQ water and the contents were mixed 
Table 1. The absorbance of different concentrations of standard gallic acid.

\begin{tabular}{cc}
\hline $\begin{array}{c}\text { Concentration } \\
(\mu \mathrm{g} / \mathrm{ml})\end{array}$ & $\begin{array}{c}\text { Absorbance } \\
(760 \mathrm{~nm})\end{array}$ \\
5 & 0.037 \\
10 & 0.069 \\
20 & 0.146 \\
30 & 0.24 \\
40 & 0.3 \\
50 & 0.39 \\
60 & 0.45 \\
70 & 0.533 \\
80 & 0.58 \\
90 & 0.701 \\
\hline
\end{tabular}

vigorously and measured at $760 \mathrm{~nm}$ according to the method of Farag et al. (2014). For each concentration, three determinations were carried out and the average of the obtained absorbance was plotted versus the concentrations.

\section{Experimental animals and infection}

Laboratory-bred male, Swiss albino mice of CD-1 strain, each weighing between $18-20$ grams were used in this study. Mice were maintained throughout the study in conditioned rooms at $21^{\circ} \mathrm{C}$ and food with $24 \%$ protein content. The animal experiment was carried out according to the internationally valid guidelines and the protocol of this study was approved by a scientific research ethics committee at the TBRI. The animal experiments were performed in accordance with the TBRI committee for laboratory animals' research guidelines [Schistosome Biological Sup- ply Program (SBSP), Theodor Bilharz Research Institute (TBRI), Giza, Egypt]. Virulent Egyptian strain of S. mansoni cercariae were obtained from infected Biomphalaria alexandria snails, which were reared and maintained at SBSP, TBRI. Each mouse in infected groups was infected by subcutaneous injection with $(60 \pm 10) S$. mansoni cercariae (Liang et al., 1987).

Drugs

Silymarin (Legalon®) purchased from (Chemical Industries Development (CID), Giza, Egypt under License of: Madaus GmbH. Germany) was given orally in a dose of $750 \mathrm{mg} / \mathrm{kg} / \mathrm{day}$ (El-Lakkany et al., 2012) for 5 days/week for 8 weeks in the form of aqueous suspension in $2 \%$ Cremophor El (Sigma Chemical Co., St. Louis, MO, USA). Praziquantel @ (Praziquantel-Sedico Pharmaceutical Co. 6th of October City, Egypt) was given orally in a total dose of $1000 \mathrm{mg} / \mathrm{kg}$ divided equally on two consecutive days (Gonnert \& Andrews, 1977) during the $6^{\text {th }}$ week post infection (PI), in the form of aqueous suspension in $2 \%$ Cremophor El. C. spinosa L. methanol extract was given orally in a dose of $100 \mathrm{mg} / \mathrm{kg} /$ day (Satyanarayana et al., 2009) for 5 days/week for 8 weeks in the form of aqueous suspension in $2 \%$ Cremophor El.

\section{Experimental design}

A batch of 60 male Swiss albino mice was infected and divided into 6 groups (I-VI) and one group was uninfected (VII; served as normal control) as follows:

Group I: Received vehicle (2 \% Cremophor EL).

Group II: Received praziquantel.

Group III: Received Capparis spinosa L. extract.

Group IV: Received both Capparis spinosa L. extract and praziquantel.

Group V: Received silymarin.

Group VI: Received both silymarin and praziquantel.

Group VII: Uninfected mice were received vehicle (2\% Cremophor EL)

Table 2. Effect of C. spinosa L. and silymarine with/without praziquantel treatment on worm burden in mice infected with S.mansoni.

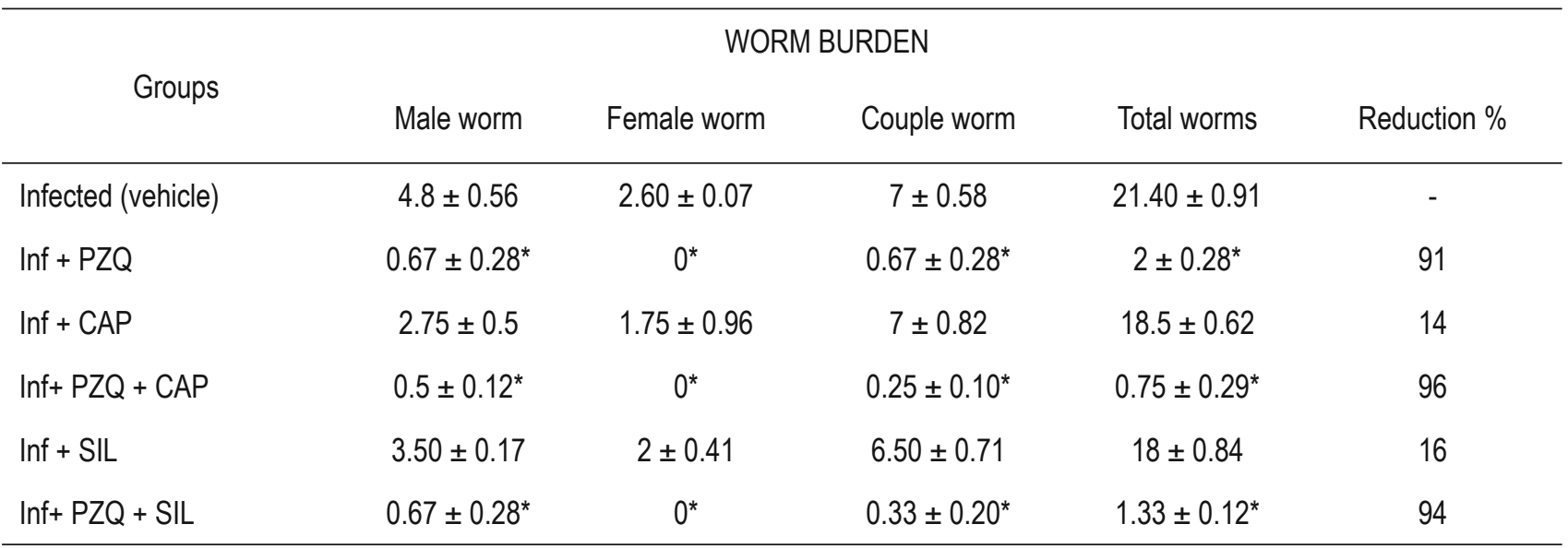

Data are presented as mean \pm SEM (standard error of the mean) ( $n=10$ in each group).

*Significantly different from infected (vehicle) group at $P<0.001$. 
Table 3. Effect of C. spinosa L. and silymarine with/without praziquantel treatment on oogram pattern in mice infected with S.mansoni.

\begin{tabular}{lccc}
\hline Groups & \multicolumn{3}{c}{ Oogram pattern (\%) } \\
& Immature ova & Mature ova & Dead ova \\
\hline Infected (vehicle) & $48.60 \pm 3.51$ & $45.20 \pm 3.27$ & $6.20 \pm 1.10$ \\
Inf + PZQ & $2.67 \pm 0.12^{*}$ & $2.33 \pm 0.80^{*}$ & $95 \pm 1.30^{*}$ \\
Inf + CAP & $39.25 \pm 2.99$ & $46.75 \pm 2.99$ & $14 \pm 1.63$ \\
Inf+ PZQ+ CAP & $0.75 \pm 0.50^{*}$ & $1 \pm 0.82^{*}$ & $98.25 \pm 1.26^{*}$ \\
Inf + SIL & $50.5 \pm 2.12$ & $40 \pm 2.54$ & $9.5 \pm 2.12$ \\
Inf+ PZQ + SIL & $1 \pm 0.33^{*}$ & $0.67 \pm 0.18^{*}$ & $98.33 \pm 1.53^{*}$ \\
\hline $\begin{array}{l}\text { Data are presented as mean } \pm \text { SEM }(\mathrm{n}=10 \text { in each group }) \\
\text { * Significantly different from infected (vehicle) group at } \mathrm{P}<0.001\end{array}$ & &
\end{tabular}

All mice were sacrificed 8 weeks from day zero experiment by decapitation. After decapitation, blood samples collected in centrifuge tubes were centrifuged at $3000 \mathrm{rpm}$ for $20 \mathrm{~min}$. Serum was stored at $-20{ }^{\circ} \mathrm{C}$ until used for biochemical assays. Porto-mesenteric perfusion was performed for recovery of $S$. mansoni worms in infected mice for subsequent counting (Duvall \& De Witte, 1967). Pieces of intestine and liver were digested in $5 \% \mathrm{KOH}$ (potassium hydroxide) solution and number of eggs per gram intestine or liver was calculated to determine the ova count in tissue (Cheever, 1968). The percentage of different egg developmental stages (Oogram pattern) were studied according to the method of Pellegrino et al. (1962) in which eggs at different stages of maturity were identified and counted.

Assessment of toxicity and determination of $L D_{50}$ A group of 18 adult ( 6 weeks old) normal male CD-1 Swiss albino mice weighing between $20-25 \mathrm{~g}$ were used to study the toxicity effect and to determine the $L D_{50}$ of $C$. spinosa extract. They were subdivided into six subgroups of three mice each. Subgroups were treated orally with increasing doses of 100, 500, 1000, 2000, 3000 and $4000 \mathrm{mg} / \mathrm{kg}$ plant extract re-suspended in $2 \%$ Cremophore EL (Sigma/Aldrich Chemical Co., St. Louis MO, USA). Mortality rates were recorded 24 hours post treatment. The death rate was used to judge toxicity.

\section{Assessment of biochemical parameters}

The Biodiagnostic kits at Dokki laboratory (Giza, Egypt) were used for the determination of serum aminotransferase enzymes (AST and ALT) activities, ALP and serum albumin.

Histopathology and Granuloma Measurement

Livers recovered from mice were fixed in $10 \%$ buffered formalin

Table 4. Effect of $C$. spinosa L. and silymarine with/without praziquantel treatment on tissue egg load in mice infected with S.mansoni.

Tissue egg load/gm $\times 10^{3}$

Groups Hepatic tissue Intestinal tissue

\begin{tabular}{lcccc} 
& Egg load & Reduction $\%$ & Eggload & Reduction \% \\
\hline Infected (vehicle) & $29.69 \pm 0.31$ & - & $31.61 \pm 0.72$ & - \\
Inf + PZQ & $5.79 \pm 0.83^{*}$ & 80 & $0.98 \pm 0.36^{*}$ & 97 \\
Inf + CAP & $16.26 \pm 0.86^{*}$ & 45 & $12.17 \pm 0.95^{*}$ & 62 \\
Inf + PZQ + CAP & $1.06 \pm 0.36^{*}$ & 96 & $0^{*}$ & 100 \\
Inf + SIL & $19.62 \pm 0.53^{*}$ & 34 & $8.63 \pm 0.30^{*}$ & 30 \\
Inf + PZQ + SIL & $0.50 \pm 0.38^{*}$ & 98 & $0^{*}$ & 100
\end{tabular}

Data are presented as mean \pm SEM $(n=10$ in each group)

* Significantly different from infected (vehicle) group at $\mathrm{P}<0.001$. 
and processed to paraffin blocks. Sections (4 $\mu \mathrm{m}$ thick) were cut $250 \mu \mathrm{m}$ away from the preceding sections to avoid measurement of the same granuloma. Five paraffin liver sections were prepared from each animal and stained with haematoxylin and eosin (H\&E) and Masson trichrome stains. Measurements of the granuloma size were conducted on non-contiguous granulomas, each containing a single egg (with intact or degenerated miracidia), using an ocular micrometer. The mean diameter of each granuloma was calculated by measuring two diameters of the lesion at right angles to each other (Von Lichtenberg, 1962). For each mouse, 40 granulomas were measured and associated hepatic histopathological changes were studied.

\section{Statistical Analysis of Data}

Data were reported as mean counts \pm standard deviation. Statistical analyses were done using computerized statistical software program IBM SPSS 19.0. The independent sample t-test was used to assess the statistical significance of the difference between two study group means. Statistical significance was defined as $P$ values $<0.05$.

\section{Results}

Results of quantitative estimation of polyphenols content using colorimetric assay

The absorbance of different concentrations of standard gallic acid was illustrated in Table 1. The present study revealed that C.spinosa $\mathrm{L}$. leaves contain higher concentration of phenolic compounds $(28.4 \mu \mathrm{g} / \mathrm{ml})$ than fruits $(7.3 \mu \mathrm{g} / \mathrm{ml})$.

Parasitological results

Praziquantel resulted in the highest percentage of worm burden reduction $(91 \%)$ when administered alone to mice infected with $S$. mansoni. This percentage increased to (96\%) and (94\%) after the synergistic effect of silymarin and $C$. spinosa extract, respectively. Infected animal groups treated with praziquantel alone, praziquantel combined with $C$. spinosa extract and praziquantel combined with silymarin extract showed significantly different effects on worm burden when compared with infected group (i.e male worm, female worm, coupled worm and total worms, at $P<0.001$ ). Generally female worms were more affected by drugs used than male worms. They died when treated with praziquantel alone, or combined with $C$. spinosa extract and silymarin extract. Meanwhile the administration of $C$. spinosa extract alone or silymarin extract alone to the $S$. mansoni infected animals yielded in the lowest worm burden reduction, $14 \%$ and $16 \%$ respectively. The effect of C. spinosa L. and silymarin with/without praziquantel on the worm burden of mice infected with S.mansoni is shown in Table 2.

All egg stages (immature, mature and dead) were observed in the intestines of infected non-treated and treated mice. As presented in Table 3, theywere evidently affected by administration of praziquantel alone and showed a significant reduction in the percentage of dead ova (95 $\pm 1.30 \%)$ when compared with groups ad- ministered with $C$. spinosa extract alone $(14 \pm 1.63 \%)$ or silymarin extract alone $(9.5 \pm 2.12 \%)$.

Administration of praziquantel alone to mice infected with S. mansoni showed the highest percentage in reduction of ova $(80,97 \%)$, what was followed by $C$. spinosa extract alone $(45,62 \%)$ and silymarin extract alone $(34,30 \%)$ in both liver and intestinal tissues, respectively. The reduction percentage of ova count increased to $100 \%$ in intestinal tissues after administration of praziquantel combined with C. spinosa extract or praziquantel combined with silymarin extract. Also, this reduction percentage in ova count was observed in hepatic tissues when treated with both praziquantel and C. spinosa extract (96\%), or praziquantel and silymarin extract $(98 \%)$. These effects of C. spinosa L. and silymarin with/ without praziquantel on tissue egg load in mice infected with $S$. mansoni are presented in Table 4.

\section{Acute Toxicity and Determination of $L D_{50}$}

Zero mortality wase observed 24 hours, as wellas post treatment with increasing doses of C. spinosa extract starting from $100 \mathrm{mg} /$ $\mathrm{kg}$ to $4000 \mathrm{mg} / \mathrm{kg}, \mathrm{LD}_{50}>4000 \mathrm{mg} / \mathrm{kg}$. That means that C. spinosa $\mathrm{L}$. extract is safe at the treatment dose of $100 \mathrm{mg} / \mathrm{kg}$.

Biochemical Parameters Assessments

Infection of mice with $S$. mansoni resulted in defective excretion and elevation of serum AST, ALT and ALP values due to livers damag. Also a serious decrease in serum alumin was observed. These results were significantly different from uninfected (vehicle) group . Administration of praziquantel, C. spinosa extract and silymarin extract either alone or in combination with praziquantel to the infected animals with $S$. mansoni showed an improvement in all liver functions which were significantly different when compared with infected (vehicle) group $(P<0.05)$. Except liver albumin the Infected animal group treated with praziquantel alone showed slightly better improvement in all tested liver functions when compared with other groups treated with $C$. spinosa extract alone or silymarin extract alone, The best results of serum albumin level were obtained when praziquantel combined with $C$. spinosa extract $(3.36 \pm 0.04 \mathrm{gm} / \mathrm{dl})$. This result was nearly the same of that of the uninfected animal group ( $3.47 \pm 0.15 \mathrm{gm} / \mathrm{dl})$, Table 5 summarizes all these results.

\section{Histopathological Study}

Stained liver sections with haematoxylin and eosin (H\&E) and Masson's trichrome (MT) of S. mansoni infected not treated mice demonstrated that liver tissue was studded with granulomas, many granulomas were fused together with intact eggs, also ballooned hepatocytes were diffusely seen. Hepatic tissues of the infected mice treated with $P Z Q$ display moderate degree of improvement with presence of many granulomas, loose fibrous reaction, less inflammatory aggregates and vasculature showed congestion together with the focal necrotic area. Hepatic tissues of infected mice treated with $C$. spinosa $L$. display mild to moderate degree of improvement, most granulomas appeared with dead egg, less fi- 

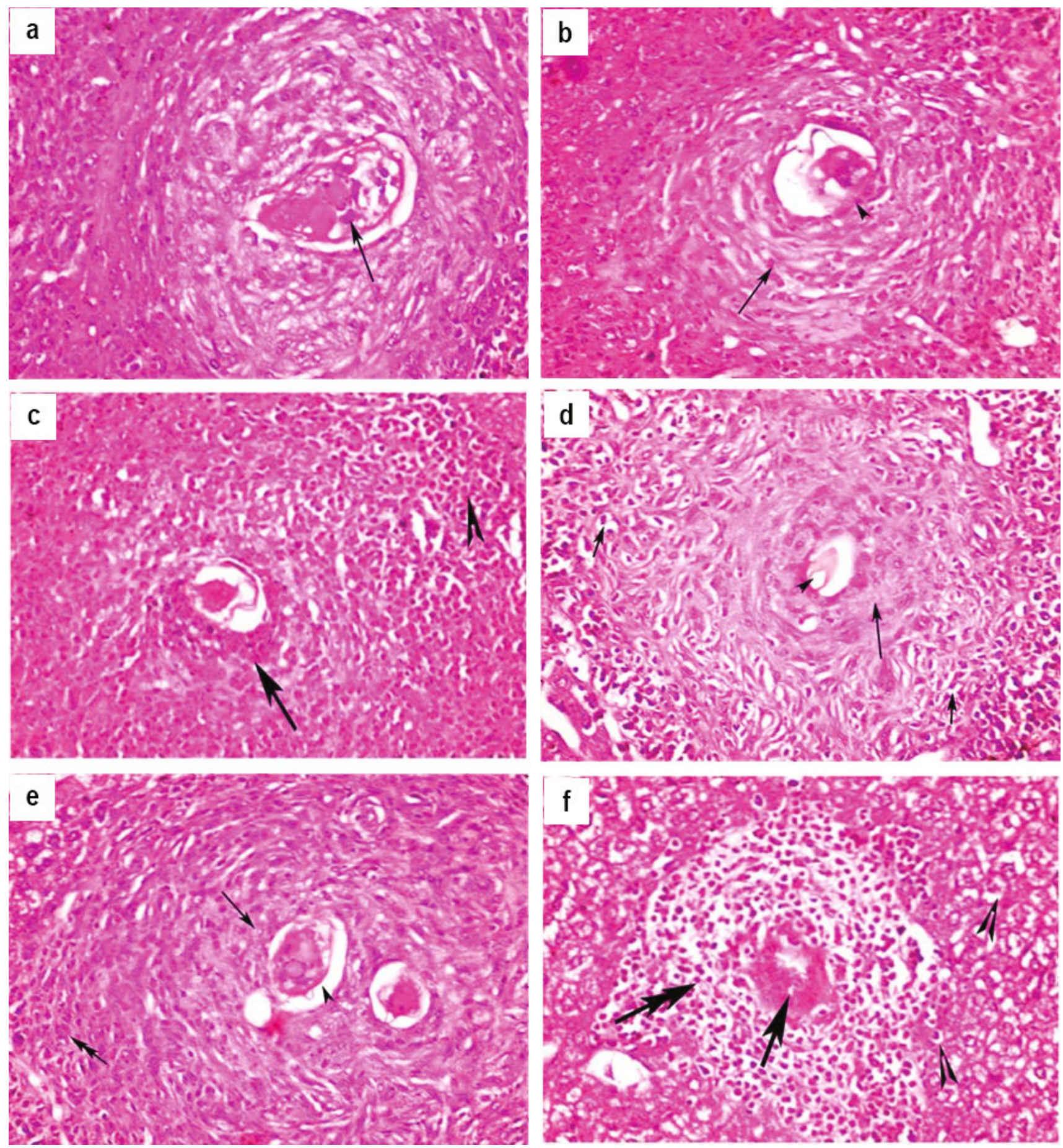

Fig. 1. Histopathological examination of haematoxylin and eosin stained liver sections (a) Infected control group demonstrating large fibrocellular granuloma surrounding one intact egg (arrow) and peripheral zone of inflammatory cells, (b) Infected mice treated with PZQ demonstrating granuloma with loose fibrotic reaction (arrow) and dead egg (arrow head), (c) Infected mice treated with C. spinosa L. extract demonstrating notable reduction in granuloma size, with loosening of the central fibrous area of granulomatous reaction (arrow) and dense inflammatory cellular response (arrow head), (d) Infected mice treated with C.spinosa L. extract and PZQ demonstrating moderate loose of fibrotic reaction (arrow), dead egg (arrow head) and less cellular inflammatory infiltrate around (short arrow), (e) Infected mice treated with silymarin demonstrating compact granuloma with dense fibrotic reaction (arrow), intact egg (arrow head) and inflammatory area (double arrow), (f) Infected mice treated with silymarin and PZQ focusing on granuloma with dead egg (arrow), loose inflammatory infiltration (double arrow), hepatocyte with vacuolated cytoplasm (arrow head) (x: 400). 

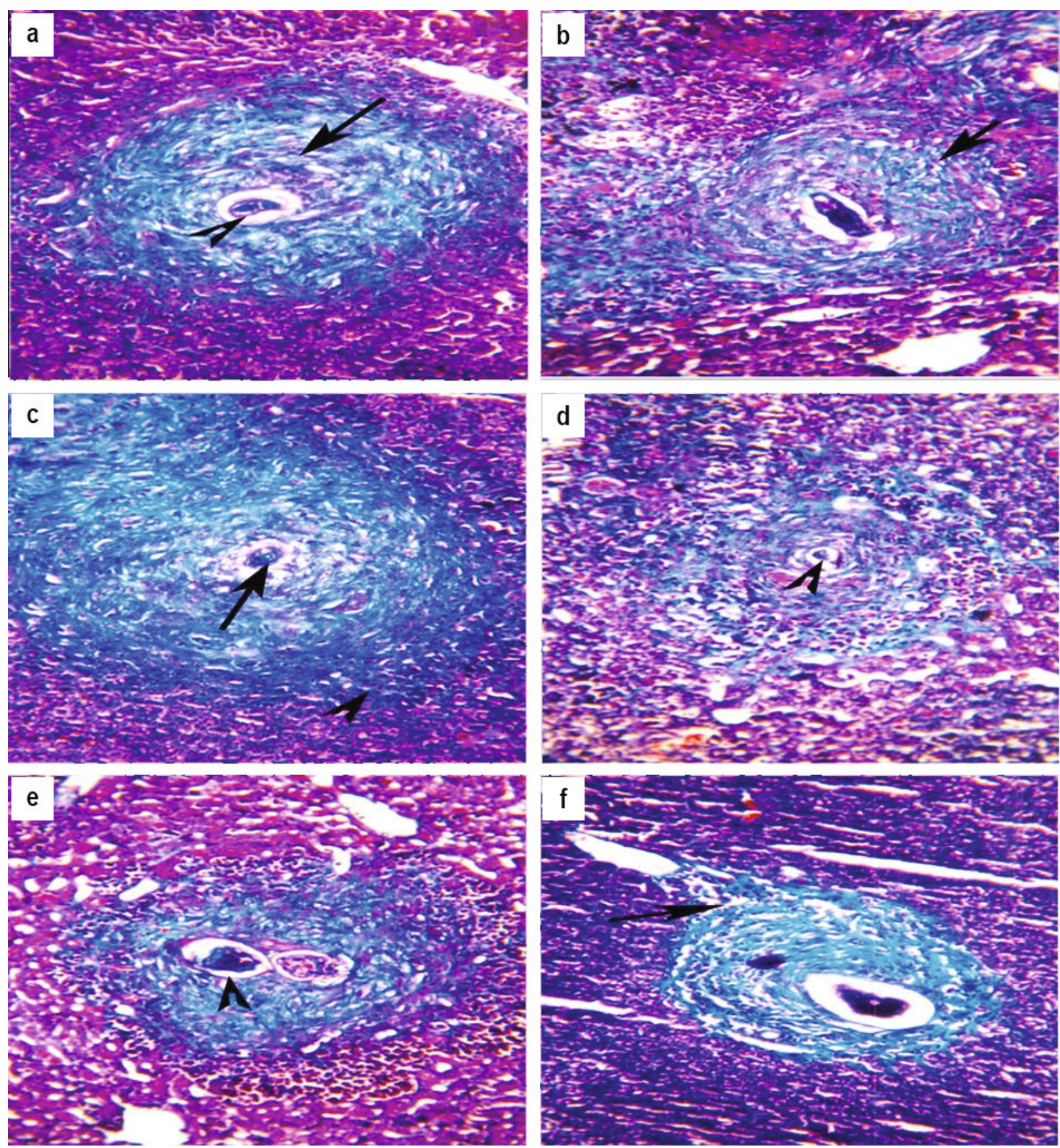

Fig. 2. Histopathological examination of liver sections from (a) Infected control group demonstrating granuloma with compact fibrous reaction (arrow) intact egg (arrow head) and moderate inflammation, (b) Infected mice treated with PZQ demonstrating loose fibrous area and mild degree of inflammatory reaction (arrow),

(c) Infected mice treated with C. spinosa L. extract demonstrating granuloma with loose fibrosis, dead egg (arrow) and external layer of inflammatory cells (arrow head),

(d) Infected mice treated with C.spinosa L. extract and PZQ demonstrating granuloma with loose fibrotic reaction and dead egg (arrow), (e) Infected mice treated with

silymarin demonstrating granuloma with intact egg (arrow head) and compact surrounding granuloma, (f) Infected mice treated with silymarin and PZQ demonstrating loose of fibrous area around dead egg and less inflammatory reaction (arrow) [Masson's Trichrome x: 400]. 
Table 5. Effect of C. spinosa L. and silymarine with/without praziquantel treatment on liver function tests 8 weeks post S.mansoni mice infection.

\begin{tabular}{lcccc}
\hline Animal Groups & Serum AST (U/L) & Serum ALT (U/L) & Serum Albumin (gm/dl) & Serum ALP (U/L) \\
\hline Uninfected (vehicle) & $42.67 \pm 0.52$ & $26.33 \pm 1.53$ & $3.47 \pm 0.15$ & $32.33 \pm 1.15$ \\
Infected (vehicle) & $111.8 \pm 2.86^{*}$ & $53.8 \pm 0.38^{*}$ & $1.9 \pm 0.27^{*}$ & $87 \pm 1.41^{*}$ \\
Inf + PZQ & $70.83 \pm 0.64^{* *}$ & $39.4 \pm 1.09^{* *}$ & $2.94 \pm 0.11^{* *}$ & $59 \pm 1.43^{* *}$ \\
Inf + CAP & $81.5 \pm 1.11^{* *}$ & $45.5 \pm 1.29^{* *}$ & $3.03 \pm 0.51^{* *}$ & $65.75 \pm 1.06^{* *}$ \\
Inf + PZQ + CAP & $62.25 \pm 1.59^{* *}$ & $30.75 \pm 0.84^{* *}$ & $3.36 \pm 0.04^{* *}$ & $48.75 \pm 1.71^{* *}$ \\
Inf + SIL & $89.5 \pm 0.71^{* *}$ & $49.75 \pm 0.35^{* *}$ & $2.75 \pm 0.07^{* *}$ & $73 \pm 1.14^{* *}$ \\
Inf + PZQ + SIL & $67.17 \pm 0.76^{* *}$ & $35.5 \pm 0.5^{* *}$ & $3.1 \pm 0.5^{* *}$ & $54.67 \pm 1.08^{* *}$ \\
\hline
\end{tabular}

Data are presented as mean \pm SEM $(n=10$ in each group).

ALT, Alanine aminotransferase; AST, Aspartate transaminase; ALP, Alkaline phosphatase

${ }^{*}$ Significantly different from uninfected (vehicle) group at $P<0.05$. ${ }^{* \star}$ Significantly different from infected (vehicle) group at $P<0.05$.

brosis, peri-inflammatory layers, and hepatocytes revealed moderate cytoplasmic vacuolation with vesicular nuclei or marginated chromatin. Hepatic tissues of infected mice treated with C. spinosa $\mathrm{L}$. and $\mathrm{PZQ}$ revealed mild to moderate ameliorating impact, where many granuloma seen with loose reaction and dead eggs, a notable reduction in the number of granuloma and patchy areas of hepatic cells showed vacuolated cytoplasm. Silymarin alone has no effect on schistosoma granuloma, but the most effective impact was on hepatocytes where wide areas of intact hepatocytes were detected. However, ballooned hepatocytes in pericentral areas could be observed together with an increase in the extracellular matrix. Hepatic tissues of infected treated mice with PZQ and silymarin showed a notable reduction in the number of granulomas and hepatic cells showed loose mild vacuolated cytoplasm as shown on Figs. 1 and 2. The number and diameter of hepatic granulomas in different experimental groups are shown on Table 6.

\section{Discussion}

Medicinal plants are used worldwide for centuries to promote and maintain health as well as to relief symptoms in chronic diseases. Polyphenols are phytochemical compounds found in plants which apparently have vital health benefits and prevent from health dangers such as cancer, high blood pressure, heart disease or diabetes (Rose, 2013). In general they are potent antioxidants, metal chelators with anti-inflammatory, antiallergic, hepatoprotective, antithrombotic, antiviral, and anticarcinogenic activities (Tapas et al., 2008).

In the present study, a quantitative estimation of total polyphenolic content using colorimetric assay revealed that $\mathrm{C}$. spinosa $\mathrm{L}$. leaves contain higher concentration of polyphenols than fruits, so the methanol extract of the leaves to estimate its biological activity was used.

Table 6. Effect of C. spinosa. L. and silymarine with/without praziquantel treatment on hepatic granuloma number and diameter 8 weeks post S.mansoni mice infection.

\begin{tabular}{lcc}
\hline Groups & $\begin{array}{c}\text { Mean number of granuloma in } 10 \\
\text { successive power fields }\end{array}$ & $\begin{array}{c}\text { Mean granuloma diameter } \\
\text { in } \mu \mathrm{m}\end{array}$ \\
\hline Infected (vehicle) & $11.63 \pm 1.52$ & $347.34+17.24$ \\
Inf + PZQ & $5.26 \pm 1.26^{*}$ & $231.56 \pm 23.34^{*}$ \\
Inf + CAP & $7.33 \pm 2.12^{*}$ & $239.34 \pm 14.45^{*}$ \\
Inf + PZQ + CAP & $4.29 \pm 1.34^{*}$ & $212.74 \pm 24.54^{*}$ \\
Inf + SIL & $6.14 \pm 1.14^{*}$ & $234 \pm 16.62^{*}$ \\
Inf + PZQ + SIL & $3.25 \pm 0.12^{*}$ & $207 \pm 13.34^{*}$ \\
\hline
\end{tabular}

Data are presented as mean \pm SEM ( $n=10$ in each group).

* Significantly different from uninfected (vehicle) group at $\mathrm{P}<0.05$ 
These results matched with the studies carried out by $Y u$ et al. (2006) who isolated some phenolic compounds from the fruit of C. spinosa L. by chromatographic methods. Also Kulisic-Bilusic et al. (2012) evaluated the aqueous infusion of caper and showed an interesting compositional pattern containing several phenolic compounds reflecting the potential benefits of the plant.

In this study, the evaluation of parasitological parameters was done to confirm that all mice had received unbiased $S$. mansoni infection. In addition the presence of hepatic granulomas around trapped schistosome eggs affecting hepatocellular functions was examined to confirm the eradication of the worms and eggs by PZQ. Histopathological examinations of hepatic sections of sacrificed mice in all groups, by staining with haematoxylin and eosin and Masson's trichrome stains, for detection of the histopathological changes and fibrosis degree were done to assess the cure rates and degree of liver tissue healing after the treatment.

In this research, treatment of $S$. mansoni infected mice with PZQ resulted in a $91 \%$ reduction in worm burden. This was accompanied with significant reduction in the hepatic and intestinal tissue egg load by $80 \%$ and $70 \%$, respectively. There was also a significant increase in the percentage of dead eggs when compared with the infected untreated group. These results are consistent with several previous studies accomplished by Issa (2007), Abdel-Rahman (2009), El-Sisi et al. (2011) and El-Lakkany et al. (2012). The main justification for these results is that $P Z Q$ affects adult schistosomes directly. This leads to their contraction, paralysis and subsequent shift from mesenteric to hepatic veins where they are finally damaged by the phagocytic cells. In addition, PZQ causes destruction of the worm tegument leading to the exposure of tegumental antigen, which becomes easily accessible to the parasites' specific antibody (Fallon et al., 1995). Administration of PZQ showed a significant improvement in all hepatic functions and associated histopathology with a significant decrease in the number and diameter of granulomas. These findings are in agreement with El-Sisi et al. (2011) and El-Lakkany et al. (2012).

Remarkably, treatment of $S$. mansoni infected mice with C. spinosa $\mathrm{L}$. alone resulted in reduction in worm burden by $14 \%$, which was accompanied with a statistically significant reduction in hepatic tissue egg load by $45 \%$ and a statistically significant reduction in intestinal tissue egg load by $62 \%$ when compared to the infected untreated group.

In addition, administration of $C$. spinosa $L$. resulted in some healing of hepatic granulomatous lesions what was supported parasitologically by partial increase in the percentage of dead ova and associated histopathology by decrease in diameter of granulomas, more ova degeneration and fewer inflammatory cells. This may be attributed to the anthelmintic, anti-inflammatory, antioxidant, antihistaminic, immunomodulatory, and antihepatotoxic activities of C. spinosa (Manikandaselvi et al., 2016). Thus, it is possible that C. spinosa L. eliminates the products of oxidative reactions and assists in the immune-mediated destruction of worms and eggs. $P Z Q$ is an active anti-schistosomal drug, against all forms of schis- tosomiasis (Ali, 2011). To stimulate further PZQ healing outcome, which could diminish liver fibrosis simultaneously with worm removal. it was recommended to develop a combination of $P Z Q$ and an antifibrotic drug in the treatment of murine schistosomiasis (Doenhoff et al., 2002). Co-administration of C. spinosa L. with $P Z Q$ also showed a statistically significant decrease in worm burden (96\%) and no viable eggs were present, associated with reduction in hepatic tissue egg load by $96 \%$ and $100 \%$, respectivealy. The reduction of intestinal tissue egg load and better healing of hepatic granulomatous lesions indicates that the use of $C$. spinosa $\mathrm{L}$. along with $\mathrm{PZQ}$ did not interfere or affect the antischistosomal effect of $P Z Q$.

The administration of $C$. spinosa $L$. either alone or in combination with praziquantel showed a statistically significant reduction in the elevated liver enzymes ALT, AST and ALP. This reduction in serum transaminases (AST and ALT) and ALP values reveals that $C$. spinosa $\mathrm{L}$. has a hepatoprotective activity. These protective effects might be as a result of plasma membrane stabilization and preservation the structural integrity of cells as well as repair of hepatic tissue damage caused by S. mansoni infection. Also, there was a significant elevation of the level of serum albumin.

Determination of enzyme levels, like serum AST and ALT is essential for the evaluation of liver damage by schistosomal infection (Aly \& Mantawy, 2013 and Al-Sayed et al., 2014). Necrosis or membrane damage releases the enzymes into circulation; so, they can be measured in the serum. In agreement with the reports of Kadry et al. (2013) and Mahmoud \& Elbessoumy (2013), the raise of these enzymes in serum may be due to the hepatocytes damage caused by the parasite eggs toxins released into the circulation. In addition, Naik et al. (2011) reported that following schistosomal infection the hepatocyte membrane damage seems to be the prime culprit for the marked increase of the serum enzymes such as AST, ALT, and ALP. Also, hypo-albuminemia may be due to malabsorption resulting from damage of intestinal mucosa due to the extrusion of great numbers of eggs or could be due to diminished synthesis, which may result from parasitic injury to hepatic cells (Oliveira et al., 2009).

This study concurred with the study performed by Satyanarayana et al. (2009) who studied the hepatoprotective effect of the alcohol extract of Capparis sepiaria Linn. Capparaceae stem against carbon tetrachloride $\left(\mathrm{CCl}_{4}\right)$-induced toxicity in albino rats. The rats were administrated daily pretreatment with an alcohol extract of C. sepiaria $(100 \mathrm{mg} / \mathrm{kg})$ and the standard silymarin $(25 \mathrm{mg} / \mathrm{kg})$ orally for 7 days. The toxicant used on 7 th day was $\mathrm{CCl} 4$ at a dose of $1.25 \mathrm{ml} / \mathrm{kg}$ as $1: 1$ mixture with olive oil. The extract resulted in a significant $(p<0.01)$ reduction of the elevated levels of aspartate transaminases (AST and ALT) total bilirubin and rise of the decreased total protein level when compared with the toxic control. El-Lakkany et al., (2012) studied the effect of silymarin on S. mansoni induced liver fibrosis. It was reported that silymarin has a favorable anti-inflammatory and anti-fibrotic activity. Its application caused a partial decrease in worm burden; hepatic tissue egg 
load, reduced the elevated ALT, and when it was combined with PZQ treatment, a complete eradication of worms, eggs and relieved liver inflammation and fibrosis were observed.

Regarding silymarin treatment, it was noticed that there was no significant difference between treatments with C. spinosa L. extract or silymarin alone or in combination with praziquantel. Silymarin promotes liver health through its anti-oxidant, anti-inflammatory, anti-proliferative, and immuno- modulatory effects (Polyak et al., 2013).

The choice of $P Z Q$ and silymarin as suitable drugs used for the treatment of schistosomiasis and liver fibrosis was matched with many previous studies. Rabia et al. (2010) proved that treatment with $\mathrm{PZQ}$ alone or combined with silymarin resulted in significant reduction of parasitological parameters wherereduction in worm burden was associated with a reduction in tissue egg load and changes in the oogram pattern. These results were confirmed also by our study.

Numerous previous studies confirmed that phenolic compounds isolated from $C$. spinosa $L$. possess hepatoprotective activity. For example Gadgoli and Mishra (1999) isolated p-methoxybenzoic acid (Phenolic compound) from C. spinosa L. leaves. It possessed significant antihepatotoxic activity against carbontetrachloride and paracetamol induced hepatotoxicity in vivo and thioacetamide and galactosamine induced hepatotoxicity in isolated rat hepatocytes. Bigoniya et al. (2013) found that kaempferol (flavonoid) isolated form $C$. spinosa $\mathrm{L}$. has a potent hepatoprotective action upon $\mathrm{CCl}_{4}$ induced oxidative stress and liver toxicity in rat. The hepatoprotective effect of kaempferol can be correlated with its efficiency to normalize the levels of serum marker enzymes and enhance the antioxidant defiance status. The findings suggest that kaempferol can be used as a safe and effective alternative chemopreventive agent in the management of liver disorders.

In conclusion, C. spinosa L. leaves extract has a promising hepatoprotective and antifibrotic properties and this effect may be attributed to the relatively high contents of polyphenols. It could be introduced as a safe and effective therapeutic tool with $P Z Q$ in the treatment of schistosomal liver fibrosis. Further studies are clearly warranted to investigate the efficacy of higher doses and longer duration of treatment of $C$. spinosa $L$. leaves extract in this model of liver fibrosis, and thus establish their clinical applicability in patients with chronic liver diseases.

\section{References}

Abdel AzIz, I.Z., El-Badry, A., El-Gay, S.H. (2011): In vitro anti-schistosomal activity of Plectranthus tenuiflorus on miracidium, cercaria and schistosomula stages of Schistosoma mansoni. Res. J. Parasitol., 6: 74 - 82. DOI: 10.3923/jp.2011.74.82

Abdel-Rahman, S.A.M. (2009): Monitoring Th1 and Th2 cytokine patterns after praziquantel and mirazid treatment in experimental mansoniasis. MD. in Parasitology, Egypt, Zagazig: Zagazig University
Albanis, E., Safadi, R., Friedman, S. (2003): Treatment of hepatic fibrosis: almost there. Curr. Gastroenterol. Rep., 5: 48 - 56. DOI: 10.1007/s11894-003-0009-7

ALI, S.A. (2011): Natural products as therapeutic agents for schistosomiasis. Res. J. Med. Plant., 5: 1 - 20. DOI: 10.3923/ rjmp.2011.1.20

Al-Said, M.S., Abdelsattar, E.A., Khalifa, S.I. El-Feraly, F.S. (1988): Isolation and identification of an anti-inflammatory principle from Capparis spinosa. Pharmazie, 43(9): 640 - 641

Al-Sayed, E., Hamid, H.A., Abu El EinIn, H.M. (2014): Molluscicidal and antischistosomal activities of methanol extracts and isolated compounds from Eucalyptus globulus and Melaleuca styphelioides. Pharm. Biol., 52 (6): 698 - 705. DOI: 10.3109/13880209.2013.865240

Aly, H.F., Mantawy, M.M. (2013): Efficiency of ginger (Zingbar officinale) against Schistosoma mansoni infection during host-parasite association. Parasitol. Int., 62: 380 - 389. DOI:10.1016/j. parint.2013.04.002

Arena, A., Bisignano, G., Pavone, B., Tomaino, A., Bonina, F.P., Saija, A., Cristani, M., D'Arrigo, M. Trombetta, D. (2008): Antiviral and immunomodulatory effect of a lyophilized extract of Capparis spinosa L. buds. Phytother. Res., 22(3): 313 - 317. DOI: 10.1002/ptr.2313 Bataller, R., Brenner, D.A. (2005): Liver fibrosis. J. Clin. Invest., 115: 209 - 218. DOI: 10.1172/JCl200524282

Batanouny, K. (Ed): (2005). A Guide to Medicinal Plants in North Africa. Malaga Spain, IUCN. 64 pp.

BIGoniYa, P., Singh, C.S., ShrivastaVA, B. (2013): In vivo and in vitro hepatoprotective potential of kaempferol, a flavone glycoside from Capparis spinosa. Int. J. Pharm. Biol. Sci., 3(4): 139 - 152

Bouriche, H., Karnouf, N., Belhadj, H., Dahamna, S., Harzalah, D., SenATOR, A. (2011): Free Radical, Metal-chelating and Antibacterial Activities of Methonolic Extract of Capparis Spinosa buds. Adv. Enviro. Bio., 5(2): $281-287$

Burke, M.L., McManus, D.P., Ramm, G.A., Duke, M., Lı, Y., Jones, M.K., GoberT, G.N. (2010): Temporal expression of chemokines dictates the hepatic inflammatory infiltrate in a murine model of schistosomiasis. PLOS Negl. Trop. Dis., 4 (2): e598. DOI: 10.1371/ journal.pntd.0000598

Cheever, A.W. (1968): Conditions affecting the accuracy of potassium hydroxide digestion techniques for counting Schistosoma mansoni eggs in tissues. Bull. World Health Organ., 39: 328 - 331 Doenhoff, M.J., Kusel, J.R., Coles, G.C., Ciolı. D. (2002): Resistance of Schistosoma mansoni to praziquantel: is there a problem? Trans. R. Soc. Trop. Med. Hyg., 96: 465 - 469. DOI: 10.1016/ S0035-9203(02)90405-0

Duvall, R.H., Dewitt, W.B. (1967): An improved perfusion technique for recovering adult schistosomes from laboratory animals. Am. J. Trop. Med. Hyg., 16(4): 483 - 486. DOI: 10.4269/ ajtmh.1967.16.483

El-Hawary, S., Taha, K., Kirillos, F., Dahab, A., Saleh, N., El-Mahis, A. (2015): Molecular identification, GC/MS and antimicrobial activity of the essential oils and extracts of three Podocarpus species. 
Int. J. Pharmacog. Phytochem., 30 (2): 1360 - 1369

El-Lakkany, N.M., Hammam, O.A., El-MaAdaWy, W.H., Badawy, A.A., AIN-SHOKA, A.A., EBeID, F.A. (2012): Anti-inflammatory/anti-fibrotic effects of the hepatoprotective silymarin and the schistosomicide praziquantel against Schistosoma mansoni-induced liver fibrosis. Paras. Vect., 5: 9. DOI: 10.1186/1756-3305-5-9

El-Sisl, A., Awara, W., El-Masry, T., El-Kowrany S., El-Gharbawy R. (2011): Effects and mechanism of action of immunomodulating agents against schistosomiasis-induced hepatic inflammation and fibrosis in mice. Res. Pharm. Biotech., 3: 32 - 45

Fallon, PC., Sturrock, RF., Niang, M. Doenhoff, MJ. (1995): Short report: Diminished susceptibility to praziquantel in a Senegal isolate of Schistosoma mansoni. Am. J. Trop. Med. Hyg., 53: 61 - 62. DOI: 10.4269/ajtmh.1995.53.61

Farag, M. Mohsen, M., Heinke, R., Wessjohann, L. (2014): Metabolomic fingerprints of 21 date palm fruit varieties from Egypt using UPLC/PDA/ESI-qTOF-MS and GC-MS analyzed by chemometrics. Food Res. Inter., 64: 218 - 226. DOI: 10.1016/j.foodres.2014.06.021

Feng, X.; Lu, J.; XIN, H.; Zhang, L.; Wang, Y. TAnG, K. (2011): Antiarthritic Active fraction of Capparis spinosa $L$. fruit and its chemical constituents. Yakugaku Zasshi, 131(3): 423 - 429

Fenwick, A., Savioli, L., Engels, D., Robert Bergquist, N., Todd, M. H. (2003): Drugs for the control of parasitic diseases: current status and development in schistosomiasis. Trends Parasitol., 19: 509 - 515. DOI: 10.1016/j.pt.2003.09.005

GADGOLI, C., MISHRA, SH. (1999): Antihepatotoxic activity of $p$-methoxybenzoic acid from Capparis spinosa. J. Ethnopharmacol., 66: 187 - 190. DOI: 10.1016/S0378-8741(98)00229-3

Gonnert, R., Andrews, P. (1977): Praziquantel, a new broad-spectrum antischistosomal agent. Z Parasitenkd., 52: 129 - 150. DOI: 10.1007/BF00389899

Homeida, M.A., El Tom, I., Nash, T., Bennett, J.L. (1991): Association of the therapeutic activity of praziquantel with the reversal of Symmers' fibrosis induced by Schistosoma mansoni. Am. J. Trop. Med. Hyg., 45: 360 - 365. DOI: 10.4269/ajtmh.1991.45.360

ISSA, RM. (2007): Schistosoma mansoni: The prophylactic and curative effects of propolis in experimentally-infected mice. Rawal. Med. J., 32: $94-98$

Kadry, S.M., Mohamed, A.M., Farrag, E.M., Dalia, B., Fayed, D.B. (2013): Influence of some micronutrients and Citharexylum quadrangular extract against liver fibrosis in Schistosoma mansoni infected mice. Afr. J. Pharm. Pharmacol., 7(38): 2628 - 2638. DOI: 10.5897/AJPP12.620

Kulisic-Bilusic, T., Schmöllerb, I., Schnäbeleb, K., Siracusac, L., RuBERTOC, G. (2012): The anticarcinogenic potential of essential oil and aqueous infusion from caper (Capparis spinosa L.). Food. Chem., 132: 261 - 267. DOI: 10.1016/j.foodchem.2011.10.074

LAM, S.K., HAN, Q.F. NG, T.B. (2008): Isolation and characterization of a lectin with potentially exploitable activities from caper (Capparis spinosa). Biosci. Rep. Immed. Pub., 29(5): 293 - 299. DOI: 10.1042/BSR2008011
LIANG, Y.S., JoHn, B.I., BoYD, D.A. (1987): Laboratory cultivation of schistosome vector snails and maintenance of schistosome life cycles. In Proceeding of the 1st Sino-American Symposium, 1, pp. $34-48$

ManiKandaselVI, S., BrindHA, P. (2014): Chemical standardization studies on Capparis spinosa L. Int. J. Pharm. Pharm. Sci., 6(Suppl. 1): $47-54$

Manikandaselvi, S., Vadivel, V., Brindha, P. (2016): Review on ethnobotanical studies of nutraceutical plant: Capparis spinosa $\mathrm{L}$. (Caper). Asian J. Pharm. Clin. Res., 9: 123 - 126

Mahmoud, E.A., Elbessoumy, A.A. (2013): Effect of curcumin on hematological, biochemical and antioxidants parameters in Schistosoma mansoni infected mice. Int. J. Sci., 2: 1 - 14

Naik, S.R., Thakare, V.N., Patil, S.R. (2011): Protective effect of curcumin on experimentally induced inflammation, hepatotoxicity and cardiotoxicity in rats: evidence of its antioxidant property. Exp. Toxicol. Pathol., 63: 419 - 431. DOI: 10.1016/j.etp.2010.03.001

Oliveira, S.A., Souza, B.S., Guimaraes-Ferreira, C.A., Barreto, E.S., Souza, S.C., Freitas, L.A., Ribeiro-Dos-Santos, R., Soares, M.B. (2008): Therapy with bone marrow cells reduces liver alterations in mice chronically infected by Schistosoma mansoni. World J. Gastroenterol., 14(38): 5842 - 5850. DOI: 10.3748/wjg.14.5842 Pellegrino, J., Oliveira, C.A., Faria, J., Cunha, A.S. (1962): New approach to the screening of drugs in experimental Schistosomiasis mansoni in mice. Am. J. Trop. Med. Hyg., 11: 201 - 215. DOI: 10.4269/ajtmh.1962.11.201

Polyak, S.J., Ferenci, P., Pawlotsky, J-M. (2013): Hepatoprotective and Antiviral Functions of Silymarin Components in HCV Infection. Hepatology, 57: 1262 - 1271. DOI: 10.1002/hep.26179

Rabia, I., Nagy, F., Aly, E., Mohamed, A., El-Assal, F., El-Amir, A. (2010): Effect of treatment with antifibrotic drugs in combination with $\mathrm{PZQ}$ in immunized Schistosoma mansoni-infected murine model. J. Amer. Sci., 6: 208 - 216. DOI: 10.7537/marsjas060510.30

Rahmani, R., Mahmoodi, M., Karimi, M., Hoseinl; F., Heydari, R., SaleHI, M. Yousefl, A. (2013): Effect of hydroalcoholic extract of Capparis spinosa fruit on blood sugar and lipid profile of diabetic and normal rats. ZJRMS, 15(11): $34-38$

Rose, G. (2013): Benefits of Polyphenols. Retrieved November 19, 2015 from http://www.live-strong.com/article/173125-benefits-of-polyphenols/.

Satyanarayana, T., Devi, K., Mathews, A.A. (2009): Hepatoprotective activity of Capparis sepiaria stem against carbon tetrachloride-induced hepatotoxicity in rats. JPRHC, 1: $34-45$

Sher, H., Alyemeni, M. (2010): Ethnobotanical and pharmaceutical evaluation of Capparis spinosa L., validity of local folk and Unani system of medicine. J. Med. Plan. Res., 4: 1751 - 1756. DOI: 10.5897/JMPR10.380

Sherif, M.M., El-ShiekH, H.H.; ElaAsser, M.M., Elbadry, M.A. (2013): In vitro evaluation of antimicrobial and cytotoxic effects of Caper (Capparis spinosa). J. App. Sci. Res., 9(4): 2944 - 2950

TAPAS, A.R., SAKarkar, D.M., KaKDE, R.B. (2008): Flavonoids as Nu- 
traceuticals: A Review. Trop. J. Pharm. Res. 7: 1089 - 1099. DOI: 10.4314/tjpr.v7i3.14693

Tlili, N., Elfalleh, W., Saadaoul, E., Khaldi, A., Triki, S., Nasri, N. (2011): The caper (Capparis L.): Ethnopharmacology, phytochemical and pharmacological properties. Fitoterapia. 8293 - 101. DOI: 10.1016/j.fitote.2010.09.006

Tesoriere, L., Butera, D., Gentile, C., Livrea, M.A. (2007): Bioactive components of Caper (Capparis spinosa L.) from Sicily and antioxidant effects in a red meat simulated gastric digestion. J. Agric. Food Chem., 55: 8465 - 8471. DOI: 10.1021/jf0714113

VON LICHTENBERG, F.C. (1962): Host response to eggs of Schistoso- ma mansoni. I. Granuloma formation in the sensitized laboratory mouse. Am. J. Pathol., 41: 711 - 731

Yu, Y., GAO, H., TANG, Z., Song, X., Wu, L. (2006): Several phenolic acids from the fruit of Capparis spinosa. Asian J. Tradit. Med., 1: $3-4$

Zhou, H., Xie, C., Jian, R., Kang, J., Kang, J., Li, Y., Zhuang, C., Yang, F., Zhang, L., Lal, L., Wu, T. Wu, X. (2011): Biflavonoids from Caper (Capparis spinosa L.) Fruits and Their Effects in Inhibiting NF-kappa B Activation. J. Agric. Food Chem., 59(7): 3060 - 3065. DOI: $10.1021 /$ jf105017j 